\title{
Une jeunesse à Paris : le melting-pot à l'irlandaise de George Moore
}

\section{Fabienne Gaspari}

\section{(2) OpenEdition \\ 12 Journals}

\section{Édition électronique}

URL : http://journals.openedition.org/etudesirlandaises/3811

DOI : 10.4000/etudesirlandaises.3811

ISSN : 2259-8863

\section{Éditeur}

Presses universitaires de Rennes

\section{Édition imprimée}

Date de publication : 30 juin 2014

Pagination : 169-182

ISBN : 978-2-7535-3449-0

ISSN : 0183-973X

\section{Référence électronique}

Fabienne Gaspari, «Une jeunesse à Paris : le melting-pot à l'irlandaise de George Moore », Études irlandaises [En ligne], 39-1 | 2014, mis en ligne le 30 juin 2016, consulté le 19 avril 2019. URL : http:// journals.openedition.org/etudesirlandaises/3811; DOI : 10.4000/etudesirlandaises.3811 


\title{
Une jeunesse à Paris : le melting-pot à l'irlandaise de George Moore
}

\author{
Fabienne GASPARI \\ Université de Pau et des Pays de l'Adour
}

Résumé

George Moore débute sa carrière par un exil géographique et culturel générant un processus de déterritorialisation que sa première œuvre autobiographique décrit. Confessions of a Young Man recrée un être en perpétuel flux façonné par toutes ses rencontres avec des artistes parisiens. Cette autobiographie s'élabore sur l'intertextualité, dessine une filiation, et montre que la création se constitue en référence à un héritage absorbé et réinventé. Sa fiction, même celle dont l'« irlandicité " a été soulignée, illustre ce processus d'assimilation, avec l'utilisation de la « ligne mélodique " inspirée par Wagner et Dujardin.

Mots clés : George Moore; influences/intertextualité; autobiographie; territoire; corps imaginaire

\section{Abstract}

George Moore started his career with a cultural and geographic exile which resulted in a process of deterritorialisation described in his first autobiographic work. Confessions of a Young Man recreates a self in perpetual flux, shaped by all his encounters with Parisian artists. This autobiography is built on intertextuality, traces a lineage, and shows how creation has its basis in an inheritance which is absorbed and reinvented. His fiction, even when its "Irishness" has been underlined, also illustrates this process of assimilation, with its use of the "melodic line" coming from Wagner and Dujardin.

Keywords: George Moore; influences/intertextuality; autobiography; territory; body-imagined

"Genius is merely the power of assimilation ${ }^{1} "$

L'écrivain anglo-irlandais George Moore (1852-1933) illustre le dynamisme produit par les échanges culturels et son héritage littéraire révèle par ailleurs la force des influences européennes, plus particulièrement françaises, qui ont façonné son œuvre. Son séjour à Paris entre 1873 et 1879 est à ce titre exemplaire du franchissement des frontières géographiques, culturelles et de la créa-

1. George Moore, Evelyn Innes (1898), London, Ernest Benn, 1929, p. 137. 
tion, à partir de cette expérience, d'un imaginaire riche et multiforme reflétant la mixité. Moore a joué le rôle de pionnier et de passeur en exportant, en quelque sorte, le naturalisme de Zola et la peinture impressionniste 2 . Cette " exportation ", en direction des terres britanniques, des ferments de la littérature fin de siècle et de l'avant-garde artistique françaises figure comme une réponse à ce que bien des artistes victoriens percevaient alors comme une censure puritaine, chape de plomb pesant sur le champ artistique anglais ${ }^{3}$. La première ouvre autobiographique de Moore, Confessions of a Young Man (1886) (Moore a publié deux ouvres de fiction, A Modern Lover en 1883 et A Mummer's Wife en 1885), peut être envisagée comme un texte fondateur, portrait de l'artiste en jeune homme refusant un héritage biologique et culturel pour s'immerger dans une autre culture. Cette autobiographie ${ }^{4}$ bouscule une série de frontières, tout d'abord géographiques, mais relevant également de la tradition littéraire et de ses codes, sans oublier ce que l'on pourrait qualifier de frontières du Moi, un Moi réinventé dans l'écriture. Le concept de "déterritorialisation " ", emprunté à Gilles Deleuze, guide ici nos analyses; il est en outre envisagé en regard de la transmission, facteur essentiel dans cette métamorphose artistique.

Après une étude des métaphores employées pour décrire ce qui s'apparente à un processus d'autocréation (à partir des diverses influences artistiques françaises s'exerçant sur ce jeune anglo-irlandais), il s'agira de montrer la nature des relations intertextuelles et les jeux de réécritures proposés par Moore dans ses confessions. L'élaboration d'un corps fantasmatique, rêvé à travers un ensemble d'images assimilant l'acquisition d'un nouvel héritage culturel à un processus organique, entraîne une construction du texte proche du patchwork de citations et de références littéraires et picturales. S'il est évident que les filiations retracées dans cet écrit autobiographique posent les fondements de l'œuvre naturaliste, puis symboliste et décadente de l'écrivain, nous nous interrogerons sur la manière dont elles ont façonné la part irlandaise de sa production, plus spécifiquement le recueil de nouvelles The Untilled Field et le roman The Lake. Ou comment ces Confessions

2. On pourrait dire que Moore a introduit l'œuvre de Zola en contrebande, car l'éditeur Vizetelly a été, en 1888, condamné à la prison pour avoir fait paraître en anglais Nana, The Soil (La Terre) et Piping-Hot! (Pot-Bouille). Ses articles sur les expositions impressionnistes à Paris ont été publiés dans The Bat et ont été suivis par trois ouvrages sur ce mouvement, Impressions and Opinions (1891), Modern Painting (1893) et Reminiscences of the Impressionist Painters (1906).

3. Voir notamment le pamphlet rédigé par George Moore contre la censure exercée à l'encontre des écrivains anglais et dont le titre, Literature at Nurse or Circulating Morals, évoque les cabinets de lecture ou « circulating libraries " (Literature at Nurse or Circulating Morals : A Polemic on Victorian Censorship (1885), Sussex, The Harvester Press Limited, 1976).

4. Il est bon de préciser ici que le récit, initialement écrit à la troisième personne, relatait les aventures d'un certain Edward Dayne, persona endossée par Moore, et que c'est au cours du travail de traduction par Édouard Dujardin, en vue d'une publication dans la Revue indépendante en 1888, que Moore opta pour la première personne, assumant ainsi le mode autobiographique.

5. Gilles Deleuze, Félix Guattari, Mille plateaux, Paris, Éditions de Minuit, 1980, p. 19. 
d'un jeune anglais (titre sous lequel ce texte a été publié dans La Revue indépendante), récit d'apprentissage au cour de l'avant-garde parisienne, ont ouvert la voie à un rejet puis à une réappropriation d'une culture et d'une terre, l'Irlande, réinventée dans la fiction. Au cœur de cette réflexion se dessine alors en filigrane la figure de James Joyce, autre "Irlandais en colère ${ }^{6}$ ", pour reprendre une expression d'Hélène Cixous.

\section{Corps de cire et " estomac artistique "}

L'objectif du voyage de Moore à Paris en 1873 est affiché dans les Confessions; la mort de son père, émancipatrice au niveau financier, coïncide avec le départ d'Irlande et ses premiers pas en tant qu'artiste :

My father's death freed me, and I sprang like a loosened bough to the light. His death gave me power to create myself - that is to say, to create a complete and absolute self out of the partial self which was all that the restraint of home had permitted; this future self, this ideal George Moore, beckoned me, lured like a ghost $[\ldots]^{7}$.

La migration géographique semble être déclenchée par cette rupture fondamentale due à la mort du père et se trouve rehaussée par un autre voyage, métaphorique. La géographie occupe une place prépondérante et fait office de principe structurant dans cette ouvre autobiographique articulée autour de trois lieux, l'Irlande, Paris, puis Londres. On peut particulièrement relever le contraste culturel que le déplacement d'un territoire à un autre permet la mise en regard de la scène artistique française et de ses innovations d'un côté et, de l'autre, le rigorisme et la censure de l'ère victorienne. Au-delà de ces oppositions, l'arrivée dans un nouveau territoire vise à l'accomplissement de soi, aboutissement d'une quête identitaire entravée par les pesanteurs de la terre d'origine. Moore rejette en bloc le carcan familial et religieux, la religion catholique représentant, au même titre que la figure paternelle, une forme d'autorité aliénante. Il fréquente à Paris les ateliers de peinture, le cercle d'artistes réunis au café La Nouvelle Athènes, et rencontre entre autres Manet, Degas, Catulle Mendès et Paul Alexis.

La fonction séminale de cet écrit autobiographique, histoire d'une genèse et livre fondateur, est affirmée dans la préface : "The book is a sort of genesis: the seed of everything I have written since will be found herein" ( $\mathrm{p}$. XI). On peut le définir comme un récit des origines et un récit-origine, car il constitue le point de départ de la création d'un corpus autobiographique et d'un nouvel être, avènement et passage du

6. Hélène Cixous, L'exil de James Joyce ou l'art du remplacement, Paris, Grasset, 1968, p. 208.

7. George Moore, Confessions of a Young Man (1886), London, William Heinemann, 1928, p. 8. Toutes les références de page dans les citations renvoient à cette édition. 
jeune Anglo-Irlandais dilettante au statut d'auteur et de créateur. Le parcours artistique, les apprentissages et initiations qui le sous-tendent conduisent à une naissance, celle de l'écrivain dont elles esquissent la figure ou plutôt le corps, comme en attestent les métaphores construisant un corps fantasmé et fantasmatique. Ses frontières floues, labiles, indiquent son aptitude à la métamorphose et illustrent une forme de déterritorialisation qui accompagne, dans le registre de l'imaginaire et de l'écriture, le mouvement géographique et l'immersion dans un nouveau milieu linguistique et culturel ${ }^{8}$. Ce milieu est rêvé comme un espace matriciel, " the womb of $a$ new nationality" (p. 124), où il devient possible de se recréer.

Le recours à des images figurant un corps imaginaire dont elles esquissent les contours, nécessairement flous pour permettre ces transformations, constitue une façon de représenter l'action du milieu parisien et des influences culturelles françaises. En l'ancrant dans des processus organiques, il donne aussi de l'idée d'autogenèse, centrale dans la pratique autobiographique, une version littérale. La rupture de la filiation familiale ouvre la voie à cette autogenèse exprimée dans les métaphores corporelles décrivant des processus de transmission et d'assimilation d'un nouvel héritage acquis par le biais de lectures multiples, de rencontres et de débats avec des artistes. Le récit des Confessions s'élabore sur ces événements d'ordre culturel plutôt que sur les menus incidents du quotidien, les rencontres amoureuses, ce qui tiendrait du récit de vie. C'est bien un parcours artistique que ce récit tend à retracer, au fil duquel le " corps " artistique en gestation dans le texte autobiographique subit, tel un morceau de cire, des mutations successives liées aux variations de ses goûts et appétits. Moore s'identifie à un homme presque vierge, "an almost virgin man" (p. 15), ouvert à toutes les influences qui tour à tour le font et le défont. Les premières phrases des Confessions introduisent un être protéiforme, fait d'une substance déformable à souhait :

My soul, so far as I understand it, has very kindly taken colour and form from the many various modes of life that self-will and an impetuous temperament have forced me to indulge in. Therefore I may say that I am free from original qualities, defects, tastes, etc. What is mine, I have acquired, or to speak more exactly, chance bestowed, and still bestows upon me. I came into the world apparently with a nature like a smooth sheet of wax, bearing no impress, but capable of receiving any; of being moulded into all shapes. (p. 2)

8. Moore insiste peu sur l'acquisition d'une langue totalement nouvelle mais remarque, après avoir quitté la France, que l'anglais aurait pu devenir, s'il avait prolongé son séjour, une langue étrangère : "if I had remained two more years in France I should never have been able to identify my thoughts with the language I am now writing in, and I should have written it as an alien. " (p. 124) Henry James, lisant son premier roman A Mummer's Wife, inspiré par l'œuvre de Zola, a souligné qu'il semblait être avoir été traduit imparfaitement en anglais à partir du français : "[it] seemed to have been thought in French and inadequately translated" (cité par Adrian Frazier, George Moore 1852-1933, New Haven, London, Yale University Press, 2000, p. 108). 
La métaphore du morceau de cire, substance infiniment malléable et surface d'impression, sert l'évocation du psychisme de l'artiste, matériau modelable et protéiforme ${ }^{9}$. Le contact avec l'univers artistique parisien est pensé sur le mode de l'empreinte et du modelage, et surtout sur celui de la métamorphose.

La description des processus façonnant l'être de l'artiste s'ancre dans un imaginaire de la passivité et de l'inconscient, du hasard et de l'inexpliqué. Désacralisant les images de l'inspiration, Moore suggère une création qui serait le produit de mécanismes viscéraux, sans flamme ni étincelle, obéissant au contraire à des mouvements organiques obscurs : "This parallel of the intellect to the blind unconsciousness of the lower organs" (p. 28). Il développe donc une autre métaphore, plus organique que celle de la cire, celle de l'estomac artistique, "artistic stomach " (p. 104), associant corps et esprit ou plus exactement assimilant l'esprit à un organe caractérisé par le besoin, l'absorption, la circulation. La transmission et l'acquisition, au fondement de son développement artistique, sont alors pensées en termes d'ingestion, excrétion, et assimilation, de toutes sortes d'aliments spirituels :

Never could I interest myself in a book if it were not the exact diet my mind required at the time, or in the very immediate future. The mind asked, received, and digested. So much was assimilated, so much expelled; then, after a season, similar demands were made, the same processes were repeated out of sight, below consciousness, as is the case in a well-ordered stomach. (p. 27)

Doté d'un appareil digestif fonctionnant, selon lui, à merveille, Moore ne démystifie pas totalement les processus créatifs. S'il s'amuse ici des codes de représentation de l'invention et de la création, ceci ne l'empêche toutefois pas de relier ce travail des viscères à une activité mystérieuse et presque magique tenant aussi de l'incarnation. Ainsi la découverte de Gautier et les phénomènes concomitants d'absorption et d'assimilation sont-ils évoqués comme une incarnation, " that part of Gautier which is now incarnate in me " (p. 28).

Il faut surtout retenir de ces références à des mécanismes biologiques l'expression de la fluidité, de la métamorphose et la mise en exergue d'une identité artistique fondée sur une nature malléable et polymorphe. Les lectures, rencontres et débats au sein d'un milieu artistique riche et nouveau, permettent la constitution d'un héritage dont la transmission est pensée sous forme d'assimilation contribuant à une genèse : processus que l'écriture autobiographique met en scène et que, dans le même temps, elle accomplit. Moore se plaît à relater certaines rencontres avec ses " maîtres ", Zola, Manet, ou encore Mallarmé, se donnant ainsi une série de pères spirituels qu’il n’hésite pas à renier par la suite.

9. Cette image du cerveau vu comme une cire molle sur laquelle la sensation imprimerait son cachet rappelle les théories des philosophes sensualistes, notamment Condillac, et était plus généralement utilisée par les philosophes matérialistes du XviıI ${ }^{\mathrm{e}}$ siècle, en lien avec le concept de tabula rasa. 
S’il dépasse certains engouements vifs mais passagers pour remettre en question, l'une après l'autre, les filiations, il ne s'agit cependant pas de simples coups de foudre artistiques. En effet, il demeure marqué par ces influences successives qui, comme il l'exprime lui-même à travers l'image de la cire, laissent leurs marques et empreintes sur son psychisme, voire le pétrissent. Abandonnées, critiquées, les œuvres un temps vénérées restent pourtant, à l'état de traces.

\section{Éloge de la déterritorialisation}

Les Confessions apparaissent, en fin de compte, comme le portrait de l'auteur en lecteur ou, plus précisément, du jeune apprenti artiste anglais en lecteur de littérature française. Si la peinture y occupe une place également importante, elle semble être quelque peu éclipsée par les références à la littérature. Ce texte devient alors le lieu où la lecture s'écrit et s'inscrit, lieu de naissance d'une conscience émergeant de son immersion dans une autre culture, un autre territoire, une autre langue. Chaque lecture est découverte et révélation, étape essentielle dans la formation de l'artiste, mais chaque lecture doit être absorbée et dépassée, selon un cycle incessant que la métaphore de l'estomac, aussi triviale soit-elle, illustre bien. Moore épouse le refus de la tradition ${ }^{10}$ exprimé par nombre d'artistes à la fin du $\mathrm{XIX}^{\mathrm{e}}$ siècle. Il reçoit en héritage une culture contemporaine autre, mais s'inscrit avec elle dans un rapport impliquant la rupture permanente et répétée propre à l'avant-garde, rapport défini par Antoine Compagnon comme " glissement de la négation de la tradition vers une tradition de la négation " : "Ce n'est plus seulement avec le passé qu'il s'agit de rompre, mais du présent même qu'il faut faire table rase si l'on ne veut pas être dépassé avant même de se produire ${ }^{11}$. "Ce dépassement, en conformité avec les principes d'audace et de provocation, s'effectue par la pratique de la citation, l'enchâssement et l'autoréférentialité, entre pastiche et parodie. Il pose également la question de l'intertextualité.

L'incipit, on l'a vu, trace les contours d'un être protéiforme, en perpétuelle métamorphose, et ouvre le texte sur une multitude de devenirs suggérés par la métaphore de la cire. Il apparaît comme un tribut payé à Walter Pater (cité dans la préface) et à son éloge de la fluidité qui, intégrant les avancées de la psychologie préfreudienne et les théories de Darwin, se fonde sur l'idée d'une mouvance et d'une multiplicité au cœur de l'être. On trouve ce même éloge chez Wilde

10. Dans Les cinq paradoxes de la modernité, Antoine Compagnon rappelle que la tradition est, selon l'étymologie, "la transmission d'un modèle ou d'une croyance, d'une génération à la suivante et d'un siècle à l'autre : elle suppose l'allégeance à l'autorité et la fidélité à une origine" (Paris, Édition du Seuil, 1990, p. 7). À la lueur de cette analyse, il est clair que Moore s'inscrit dans la rupture.

11. Ibid., p. 54. 
(influencé lui aussi par les écrits de Pater), où il participe de l'éthique et de l'esthétique du Dandy :

He used to wonder at the shallow psychology of those who conceived the Ego in man as a thing simple, reliable, and of one essence. To him, man was a being with myriad lives and myriad sensations, a complex multiform creature that bore within itself strange legacies of thought and passion, and whose very flesh was tainted with the monstrous maladies of the dead $[\ldots]^{12}$.

Que les pensées de Dorian soient formulées alors qu'il se trouve sous l'emprise de la lecture du fameux livre jaune ( $\grave{A}$ rebours de Huysmans) illustre à merveille le phénomène d'échange entre l'Angleterre et la France. Si elles rappellent bien évidemment le lien entre l'héritage et l'hérédité pensée comme " revenance $^{13}$ " et prenant une résonance gothique au $\mathrm{XIX}^{\mathrm{e}}$ siècle (le retour d'un passé biologique réincarné dans la descendance), ces réflexions font de l'être humain un palimpseste.

Complexité, fluidité, multiplicité sont trois éléments essentiels sur lesquels se trouve articulée l'identité artistique dans Confessions of a Young Man. Or, les phénomènes de mutation et de déterritorialisation caractérisent non seulement le sujet, représenté comme protéiforme, mais aussi le récit, se démarquant de la tradition de la littérature psychologique religieuse fondée par les confessions de St Augustin. Reprenant à son compte la rupture initiée par Rousseau (que Moore n'aurait toutefois lu qu'après la rédaction de sa première version), l'auteur prend ses distances avec un texte fondateur : " $S^{t}$ Augustine's Confessions are the story of a God-tortured, mine of an art-tortured, soul» (p. 185). L'idée de l'art comme nouvelle forme de religion apparait de manière implicite dans le parallélisme des formules se faisant écho et effectuant la substitution de l'art à Dieu. Nous l'avons déjà dit, le récit fait la part belle aux événements relevant de l'apprentissage artistique, effaçant d'autres détails biographiques pour donner l'impression d'un parcours dans une bibliothèque ou un musée imaginaires. Dans sa reconstitution des filiations françaises tissées par le jeune anglais, le discours autobiographique se construit sur des bris de peinture et, avant tout, d'écriture et forme une sorte de bric-à-brac de fragments d'œuvres littéraires et picturales citées, imitées, ou commentées.

L'héritage " digéré ", selon ses propres dires, par le jeune écrivain est présenté comme un collage, un montage, au cœur d'un texte qui se fait mosaïque, image convoquée par Julia Kristeva pour définir l'intertextualité : "Tout texte se construit comme mosäque de citations, tout texte est absorption et transforma-

12. Oscar Wilde, The Picture of Dorian Gray, New York, London, Norton, 2007, p. 119.

13. Terme emprunté au titre de l'ouvrage de Jean-François Hamel, Revenances de l'histoire, Paris, Éditions de Minuit, 2006. 
tion d'un autre texte ${ }^{14}$. " Absorption et transformation : ces deux termes rappellent bien sûr le fonctionnement de "l'estomac artistique " moorien. Histoire de filiation, les Confessions n'épousent cependant pas l'architecture d'un arbre généalogique mais elles suivent une structure en rhizome, un ensemble de "lignes de fuite " ou «lignes d'erre ". Le récit, tenant de la " carte " et non du " calque $^{15}$ ", dessine bel et bien une carte des influences, se veine d'un réseau de références aux ramifications multiples, fonds littéraire où Moore vient puiser son inspiration.

Épousant le principe de remise en question des normes établies, l'auteur prend soin de se démarquer de ces références, avec lesquelles il établit des relations complexes, d'où un texte fondé sur des principes d'écart et de dépassement. Imiter implique de pratiquer le décalage et l'ironie. Les excès rhétoriques de l'autobiographe s'inscrivent en regard des excès du jeune esthète qu'il était alors, tous deux adoptant des poses : celle de l'écrivain décadent s'amusant par exemple à se déclarer " efféminé, maladif, pervers ", "feminine, morbid, perverse " (p. 48); celle du jeune Anglais, dandy se prélassant en kimono à la lueur des cierges, parmi les lys, les tentures écarlates, le parfum des gardénias, avec son python et son chat persan. Ce décor évoque, comme le montre Adrian Frazier, la demeure de Des Esseintes dans À Rebours de Huysmans : "When Moore came to describe the interior for the 1888 Confessions of a Young Man, he clearly added to its appointments some elements that he derived from Huysmans's À Rebours (1884) ${ }^{16}$." Il s'agit certes de prendre ses distances avec son moi passé, distances inévitablement creusées par le temps ${ }^{17}$, mais surtout de déconstruire, dans le même temps, le discours imité.

Dans un article sur les miniatures dans le texte de Moore, Pascale Guillot-Mac Garry étudie la dimension satirique des effets intertextuels. Les multiples poèmes insérés sont soit empruntés à des auteurs chers à Moore, soit écrits par lui-même dans une veine similaire, ce qui fait de ce jeu citationnel une forme d'exploration de l'écriture poétique ainsi mise en abyme et des Confessions " un brouillon de son œuvre à venir ${ }^{18}$ ». Guillot-Mac Garry va plus loin en montrant que, par exemple, les deux pages consacrées à un commentaire sur le crépuscule de Verlaine (p. 64-65) sont en fait une reprise, sous forme de pastiche, d'un passage d'À Rebours proposant une rêverie sur le même sujet. Moore reproduit les stratégies employées par les poètes du Cercle Zutique, créé en 1871 et rassemblé

14. Julia Kristeva, Séméiôtiké, Paris, Seuil, 1969, p. 85.

15. "Tout autre est le rhizome, carte et non pas calque " (Deleuze et Guattari, op. cit., p. 20)

16. Adrian Frazier, op. cit., p. 42.

17. Adrian Frazier montre l'effet ironique que produit un tel décalage, par ailleurs au fondement de toute entreprise autobiographique : That youthful being emerges as a figment of the ironic relationship between the unactualised self or the boy in the process of becoming and the ever more assured incarnations of the endlessly reminiscent fictionaliser "George Moore". "Myself was the goal I was making for", as he himself states the paradox of identity, and the self on show is all mirrors on mirrors. " (ibid., p. 35).

18. Pascale Guillot-Mac Garry, "Les miniatures dans Confessions of a Young Man », Cahiers irlandais, n 8, 1983, p. 11. 
autour de la figure de Cabaner dont Moore fait l'éloge sur 4 pages (p. 91-95) ${ }^{19}$. Si l'on reprend ce qu'écrit Antoine Compagnon au sujet du travail de la citation, il apparaît que l'entreprise de Moore relève de "l'appropriation ou la reprise, c'està-dire le produit de la force qui saisit la citation par le déplacement qu'elle lui fait $\operatorname{subir}^{20} »$. Cette pratique, qui produit une forme d'auto-référentialité parodique, a pour effet une déstabilisation et une réinterprétation permanentes du discours.

Refusant les formes fixes pour privilégier le mouvement et la métamorphose, l'autobiographie moorienne ne serait-elle pas alors une "hétérotopie ", espace autre et contestataire dont Foucault montre qu'il se construit sur une rupture? Le déplacement opéré par et dans l'écrit autobiographique permettrait alors de redéfinir le sujet et de le recréer ${ }^{21}$. Ce déplacement est effectué, comme nous l'avons montré, de diverses manières : par le voyage géographique, entraînant la découverte d'une autre langue et d'un autre milieu, social et culturel; par l'élaboration d'une forme littéraire, assimilée à une autogenèse, et la déconstruction systématique (métaphores organiques et jeu avec les références et citations) de toute forme codifiée, perçue comme carcan, possible entrave à la création.

\section{Retour en terre d'Irlande}

Une telle déconstruction, élaborée sur la rupture avec ses origines, semble paradoxalement conduire Moore à un retour. Après l'exil parisien, l'écrivain renoue avec ses racines, avec une culture gaélique dont il sent bien vite à nouveau les pesanteurs, et participe, pour un temps, au mouvement de renouveau culturel mené par Yeats et Edward Martyn. Il écrit des pièces de théâtre pour la scène irlandaise (The Bending of the Bough, Diarmuid and Grania) et surtout trois ouvrages de fiction s'ancrant dans sa terre d'origine, A Drama in Muslin, The Untilled Field et The Lake. Au fondement de la création moorienne et joycienne, la rébellion et l'exil, indissociables d'une fascination pour l'Irlande, sont les instruments employés pour forger une vision singulière, promise par Joyce à la fin de A Portrait of the Artist as a Young Man ("I go [...] to forge in the smithy of my soul the uncreated conscience of my race $\left.{ }^{22} »\right)$, le narrateur, Stephen, jurant d'utiliser pour seules armes le silence, la ruse et l'exil. Cette "irlandicité » est finalement,

19. L'Album Zutique contenait des parodies visant essentiellement les poètes du Parnasse. Elles étaient signées du nom de l'auteur imité, suivi du monogramme des vrais auteurs. On trouve dans Confessions of a Young Man un poème clairement dérivé de la veine scatologique et obscène développée par les zutiques : «La ballade d'Alfred, Alfred aux belles dents ", "un très grand macqillustre dans le square... " (p. 205-206).

20. Antoine Compagnon, La seconde main ou le travail de la citation, Paris, Seuil, 1979, p. 9.

21. Ces propos sont inspirés par l'article de Frédéric Regard qui emprunte à Foucault la notion d'hétérotopie pour définir les liens entre autobiographie, espace et déplacement ("L'Auteur remis en place : topologie et tropologie du sujet autobiographique ", Jacques-Lefevre N. (dir.) avec la collaboration de Frédéric Regard, Une histoire de la "fonction-auteur » est-elle possible?, Saint-Étienne, Publications de l'Université de St Étienne, 2001, p. 33-47).

22. James Joyce, A Portrait of the Artist as a Young Man (1916), Harmondsworth, Penguin Books, 1970, p. 253. 
chez George Moore comme chez James Joyce, « le produit de [leur] Irlande intérieure $^{23}$ " : Joyce dans Dubliners et Moore dans The Untilled Field ont pour projet une œuvre inédite, inspirée de leurs influences européennes; tous deux se réclament de Flaubert, Zola, Maupassant, Ibsen, Tourgueniev, ce dernier constituant la référence majeure pour l'écriture de la nouvelle. En terme d'influence stylistique, le lien avec Édouard Dujardin s'impose et nous y reviendrons. Fondées sur des détails géographiques, biographiques, historiques, et culturels, les nouvelles de Moore, comme celles de Joyce, deviennent le lieu de dramatisation des conflits entre l'écrivain et l'objet de l'écriture, l'Irlande.

Le choix du titre du recueil, The Untilled Field (publié en 1903), révèle la nature de l'entreprise de Moore, l'exploration d'un nouveau champ qu'il s'agit pour lui de défricher : une vision réaliste de la paysannerie irlandaise actuelle, débarrassée des caractéristiques mythologiques et passéistes imposées par le courant de renouveau culturel engagé par Yeats. Le champ en friche désigne le sujet littéraire original, neuf, ainsi que les terres stériles d'Irlande, désert matériel et spirituel ${ }^{24}$. Moore veut à son tour initier une tradition, transmettre un héritage et, s'il écrit ces nouvelles, "c'est afin de fournir un modèle aux futurs jeunes écrivains irlandais ", " in the hope of furnishing the young Irish of the future with models ${ }^{25} \mathrm{M}$. Avec Dubliners, Joyce, lui, veut présenter Dublin au monde, pour que la ville redevienne capitale européenne. Il apparait que Moore avait lu Dubliners avant de publier une version revue et corrigée de The Untilled Field et que Joyce connaissait les nouvelles de Moore. Au titre de la discussion sur la notion d'influence, il est bon de préciser que, malgré les critiques formulées par l'un et l'autre écrivains ${ }^{26}$, ces deux recueils constituent une illustration assez complexe de la question de l'intertextualité. Tous deux sont sous-tendus par une tension vers un ailleurs géographique, existentiel, artistique et mettent en scène l'insularité et ses avatars, enfermement, claustrophobie, paralysie. Les ruines encombrant le paysage dans The Untilled Field, "these signs of ancient Ireland " ("Home Sickness » p. 28), renvoient à une culture gaélique moribonde.

23. Hélène Cixous, op.cit., p. 15.

24. On retrouve cette association entre l'Irlande et un champ en friche dans les maximes rédigées par Joyce alors qu'il enseignait : "Ireland is a great country. It is called the Emerald Isle. The metropolitan government, after centuries of strangling it, has laid it waste. It's now an untilled field. " (cité par Richard Ellmann, James Joyce, London, Oxford University Press, 1959, p. 225)

25. George Moore, Preface, The Untilled Field (1903), Dublin, Gill and MacMillan, 1990, p. xix. Écrit en anglais par Moore, le recueil a été traduit en gaélique. Selon Moore, toujours dans la préface, il ne devait être publié que dans cette langue: "As soon as his [Tiagh Donoghue's] translations were finished, my manuscripts were to be burnt." (p. XIX).

26. S’il admirait "The Dead », Moore trouvait certaines des nouvelles de Dubliners triviales et désagréables, "trivial and disagreeable" (cité par Richard Ellmann, op. cit., p. 418). On sait, par une lettre à Stanislaus, que Joyce avait lu The Untilled Field et qu'il n'appréciait guère cette œuvre : "I read that silly, wretched book of Moore's The Untilled Field which the Americans found so remarkable for its "craftsmanship". O, dear me! It is very dull and flat indeed: and ill-written. " (Lettre à Stanislaus, 24 septembre 1905). Moore avait déjà été critiqué en 1901 par Joyce dans The Day of the Rabblement, écrit contre la culture officielle en Irlande. 
Ce qui pourrait à première vue s'apparenter à une méditation poétique sur les ruines ne renvoie au final qu'au vide au cour d'un paysage en décomposition et au cauchemar de l'histoire présente (famine, émigration, agitation sociale, entre autres maux). Cette plongée des personnages dans un passé mythique, mise en abyme de celle de Moore, se solde par un échec et un désir d'exil; constat désabusé partagé par Joyce, qui voyait dans le mouvement de renouveau irlandais une croyance naïve en un âge d'or irrémédiablement perdu. Selon Hélène Cixous, le gaélique est, pour Joyce, « la langue d'une civilisation morte. Même, elle est mortelle; émanent d'elle les charmes paralysants d'un passé légendaire et irréel, hanté de fées et de héros primitifs et superstitieux $^{27}$ ".

Refusant la tradition considérée comme un repli autarcique sur une culture insulaire stérile, épousant de nouveaux modèles littéraires européens, Moore et Joyce n'en sont toutefois pas de pâles imitateurs. Leur œuvre irlandaise apparaît comme un véritable creuset, melting pot dans lequel viennent fusionner leurs sources d'inspiration et modèles divers. Cette image est d'ailleurs devenue, aux yeux de Moore, la meilleure façon de définir la création, l’ouverture permanente à de nouvelles formes d'art, le détachement de tout dogme, tout code, toute règle : "The artist must arrive at a new estimate of things; all must go into the melting-pot in the hope that out of the pot may emerge a new consummation of himself. " Cette fusion trouve peut-être sa plus belle illustration dans l'adaptation par Moore du monologue intérieur découvert dans Les lauriers sont coupés de Dujardin : cette forme narrative expérimentale, permettant également d'exprimer l'exil intérieur vécu par les personnages, vient se fondre avec le motif fondateur de l'insularité. L'adoption du personnage comme conscience focale, le développement du monologue intérieur, reflètent cet état existentiel, l'écriture reproduisant alors dans ses formes l'insularité de la conscience d'êtres isolés ou, pour reprendre le terme employé par Richard Ellmann, "insularisés " ( islanded $^{29}$ "). Sous couvert d'une narration omnisciente à la troisième personne du singulier, discours intérieur rapporté au style indirect libre, les récits laissent deviner l'enfermement du personnage dans un désert linguistique et spirituel.

Dans The Lake (1905), cette forme expérimentale trouve pleinement sa réalisation. Elle associe la ligne mélodique ou "melodic line » et les trouvailles de Dujardin $^{30}$ qui, dans leur correspondance, conseille également Moore en matière

27. Hélène Cixous, op. cit., p. 208.

28. George Moore, Hail and Farewell, Vale (1914), London, Heinemann, 1937, p. 103-104.

29. Richard Ellmann, op. cit., p. 368.

30. Richard Allen Cave établit clairement l'influence de Dujardin sur Moore. Selon lui, Dujardin, éditeur de $L a$ Revue wagnérienne, cherchait à introduire dans le roman poésie et musique, et à adapter les principes de Wagner au récit : "but none were as avid as Dujardin in exploring ways of compelling language in poetry and the novel to yield effects analogous with Wagner's use of leitmotival musical phrases for psychological penetration and emotional intensity in his music dramas. [...] Dujardin's seminal role first lay in offering detailed thematic interpretations of 
de technique musicale, notamment sur le leitmotif wagnérien ${ }^{31}$. Texte hybride, roman par lettres entrecoupé d'un récit, cette œuvre est une réflexion sur l'écriture, l'identité, le corps et le désir, un livre-paysage ("landscape book», écrivait Moore à Lady Cunard ${ }^{32}$ ). Elle décrit un processus d'affranchissement vécu par un prêtre, grâce à un échange épistolaire avec une jeune femme, mère célibataire qu'il a chassée de sa paroisse, échange associé à un contact renouvelé avec les espaces naturels ${ }^{33}$. La ligne mélodique restitue le flux de la vie sub-consciente du personnage ou, pour citer Moore, "the essential rather than the daily life of the priest ", tissant une histoire à partir de la substance de l'âme, "the weaving of a story out of the soul's substance ${ }^{34}$ ». À travers l'immersion du prêtre dans le paysage, le récit parcourt un ensemble de lieux (île, lac, jardin, forêt) qui, devenus des sites privilégiés où s'exprime le désir tour à tour libéré ou refoulé, forment une constellation.

Le parcours géographique et culturel de la correspondante du prêtre, tout particulièrement sa découverte de Wagner, reflète les nombreuses incursions culturelles de Moore dans d'autres territoires. Ce voyage, effectué en compagnie d'un intellectuel rebaptisé Mr. Poole dans la dernière version du roman et donc explicitement associé à une forme de stagnation, apparaît cependant comme vain et stérile en regard du parcours du prêtre au cœur du paysage irlandais, espace où le sens est éclipsé et aboli. Ces errances-ci redessinent dans la diégèse un ailleurs du corps et de l'être, tandis que la ligne mélodique renvoie à un ailleurs de l'écriture cherchant à figurer le corps et le désir, tout en demeurant tendue vers cette expression idéale que serait la musique : Moore, grand admirateur de Pater, suit ici le précepte formulé dans The Renaissance. L'excipit, montrant le prêtre abandonnant ses vêtements pour se jeter dans l'eau du lac et nager, prélude à un départ vers les États-Unis, illustre l'issue de la crise relatée dans ce roman. Cette crise peut d'ailleurs être envisagée comme une illustration de ce tournant dans le roman britannique au début $\mathrm{Xx}^{\mathrm{e}}$ siècle, conduisant, selon Philip Weinstein, à une redéfini-

the operas for Moore, thereby defining Wagner's recurring preoccupations with the conflicts of sensuality with interest, of passion with religion, of Pagan with Christian ideals, of the life-force with renunciation, which Moore was to make the central subjects of his own late novels. " (A Study of the Novels of George Moore, Gerrards Cross, Colin Smythe, 1978, p. 137)

31. Sur cette correspondance, voir Michel Brunet, " "Mais qui voudrait me lire en français?” : Reading George Moore's Letters to Édouard Dujardin », Dabrigeon-Garcier F. et Huguet C. (dir.), George Moore, Across Borders, Amsterdam, Rodopi, 2013, p. 125-136.

32. George Moore, "Letter to Lady Cunard ", cité par Max E. Cordonnier, "Siegfried in Ireland : A Study of Moore's The Lake ", Welsh R. (dir.), The Way Back: George Moore's The Untilled Field and The Lake, Dublin, Wolfhound Press, 1982, p. 93.

33. Sur le lien entre écriture épistolaire, corps et paysage, voir Fabienne Gaspari, Contraintes et pesanteurs du corps dans les romans de George Moore, Thèse de Doctorat, Bordeaux III, 1998, p. 381-429. Voir également l'article de Fabienne Dabrigeon-Garcier sur le rapport dialogique entre lettres et monologue intérieur, "A Letter came into his mind": Fictional Correspondence in The Lake ", Dabrigeon-Garcier F. et Huguet C. (dir.), George Moore, Across Borders, op. cit., p. 239-253.

34. George Moore, Preface, The Lake (1905), Gerrards Cross, Colin Smythe, 1980, p. x. 
tion du personnage, "less a figure defined by semantics, signification, cultural value, and more one defined by desire, force, natural impulse ": à l'être immaculé "the immaculate self-self-withholding and self-aware", est substitué l'être incarné, "the incarnate self-sexualized and immersed beyond self-knowing in experience ${ }^{35}$ ".

Dans Confessions of a Young Man, texte fondateur d'une production autobiographique riche, dans laquelle l'artiste refuse une filiation imposée pour se recréer à partir d'influences choisies, Moore situe le lac du domaine de Moore Hall au cœur du processus de recréation de son identité :

before me the crystal lake, the distant mountains, the swaying woods, said but one word, and that word was - self; not the self that was then mine, but the self on whose creation I was enthusiastically determined. (p. 8)

Il semble que le lac, élément essentiel d'une géographie imaginaire élaborée sur une tension entre rejet et célébration, permette d'établir, sur un plan symbolique, le lien entre ce premier écrit et l'œuvre romanesque The Lake. Selon Pierre Jourde, les géographies imaginaires, fondées sur une contradiction entre présence et absence, renvoient à l'écriture même, en illustrant la recherche d'un centre absent où se produirait la coïncidence de l'être à soi :

C'est parce qu'elles représentent spatialement cette tension que les géographies imaginaires nous parlent de l'œuvre. L'écriture, séparation d'avec soi, est le rêve de la coïncidence avec soi, dans ce centre enfin retrouvé, au terme de ce parcours de « l'espace littéraire ». La géographie imaginaire nous installe au cœur de l'inexistant, elle multiplie les signes (verbaux, iconographiques) d'une présence absente, mais qui ne fait qu'exacerber et rendre plus sensible la contradiction inhérente au cœur même de l'écriture $[\ldots]^{36}$.

On sait que la seconde moitié du $\mathrm{XIX}^{\mathrm{e}}$ siècle a vu apparaître une redéfinition radicale de l'espace, du temps et de l'identité. Qu'il soit biologique ou culturel, l'héritage est repensé à l'aune du darwinisme et de la psychologie pré-freudienne. La notion de transmission est profondément modifiée, dans la mesure où le désir de modernité instaure une relation complexe à la tradition et génère une tradition de la rupture, tout en mettant en exergue l'image du palimpseste. Moore propose son interprétation de ce " devenir autre ${ }^{37}$ », processus déclenché par la déterritorialisation géographique et culturelle vécue par le jeune Anglo-Irlandais en exil. Ses Confessions, où il se rêve comme un morceau de cire, font l'éloge du déplacement, de la fluidité, de la métamorphose. Son ouvre irlandaise offre, elle, un creuset

35. Philip Weinstein, The Semantics of Desire, Princeton, Princeton University Press, 1984, p. viI.

36. Pierre Jourde, Géographies imaginaires, Paris, José Corti, 1991, p. 314.

37. Gilles Deleuze, Félix Guattari, op. cit., p. 291. 


\section{Fabienne Gaspari}

dans lequel viennent se fondre les influences européennes et où se crée une géographie imaginaire. Bien que cet écrivain se soit vu reléguer, selon Jean-Claude Noël, auteur d'une biographie parue en 1966, " dans le purgatoire des réputations littéraires ${ }^{38}$ ", cette œuvre constitue une ébauche de la littérature moderniste anglaise et annonce le flux de conscience qui traverse les romans de James Joyce et de Virginia Woolf.

38. Jean-Claude Noël, George Moore : l'homme et l'œuvre (1852-1933), Paris, Didier, 1966, p. 551. 